\title{
Belegarzttarif
}

\author{
H. H. Brunner, Präsident FMH
}

An dieser Stelle wurde wiederholt ausgeführt, dass Belegärzte eine entscheidende Rolle für die Versorgung unserer Bevölkerung mit qualitativ hochstehenden und kosteneffizienten Leistungen spielen. Diese Position wurde zu Zeiten nicht von allen Parteien des schweizerischen Gesundheitswesens mitgetragen, insbesondere nicht von anderen Vertragsparteien TARMED. Es ist erfreulich, dass die Versicherer hier über die Bücher gegangen sind ... wohl auch gehen mussten, weil die Anwendung von TARMED vor allem im Bereiche KVG zu einem eigentlichen Exterminationsprogramm von operativ Tätigen bzw. Belegärzten auszuarten droht, verschärft noch durch die dauernde Taxpunktwertabsenkung, die nach sich langsam konkretisierender Erkenntnis vor allem jene treffen wird, die die Kostensteigerung gar nicht zu verantworten haben.

Es ist unbestritten, dass die Tätigkeit des Belegarztes in den TARMED-Tarifstrukturen nicht abgebildet sind - prosaisch formuliert: nicht adäquat bezahlt werden. Eine Feststellung, die seitens der FMH seit 1993 (!) konstant vertreten wurde und wird. Die Versicherer bieten nun Hand, gleichsam ex post diese Strukturdefizite zu korrigieren. Der Vorgehensplan sieht in etwa so aus.

\section{Etappe: 2004}

\section{UV/MV/IV}

- Schaffung einer sogenannten arztnahen TLKomponente, die nicht nur besondere infrastrukturelle Aufwendungen abgilt, die vom Belegspital nicht bezahlt werden, sondern insbesondere die Fixkosten bzw. Sprungfixkosten der «leerstehenden» belegärztlichen Praxis während seiner Abwesenheit für seine belegärztliche Tätigkeit. Diese arztnahe TL wird pro Minute belegärztlicher Tätigkeit bezahlt und liegt nach Stand der Verhandlungen im Bereiche von Fr. 2.29; vertraglich und tarifmechanisch ist sichergestellt, dass der Belegarzt und nicht das Spital diese Abgeltung erhält. Der definitive Vertrag wird in der Schweizerischen Ärztezeitung publiziert werden. Die Belegarzttätigkeit wird als separate Entität im Rahmen der Fallkostenstabilität während 18 Monaten analysiert und ge- steuert, analog den im UV/MV/IV-Bereich angewendeten Vorgehensweisen und Prinzipien unter Verantwortung einer von MTK/ FMH-SBV bestimmten Kommission. Damit ist sichergestellt, dass die belegärztliche TL nicht im Rahmen zu erwartender Turbulenzen um die Fallkostenstabilität in Spitälern durch grossräumige TPW-Anpassungen «phagozytiert» wird;

- Voraussetzung für die Abgeltung ist der Beitritt zum UV/MV/IV-Vertrag mit der FMH vom 28. Dezember 2002 sowie einem gemäss diesen Punktationen erstellten Übergangsvertrages für Belegärzte;

- die Diskussionen mit den Krankenversicherern wurden angesponnen, eine ähnliche Vereinbarung auch im KVG-Bereich treffen zu können. Die Zeichen für eine erfolgreiche Vereinbarung sind günstig.

\section{Etappe: 2005}

Bis 31. Dezember 2005 soll der Belegarzttarif fertiggestellt werden. Dieser

- baut auf TARMED auf, löst das Problem Assistenzarzt und - definitiv - die Abgeltung der belegärztlichen Infrastruktur;

- aggregiert die Einzelleistungen zu diagnosebezogenen Pauschalen;

- lässt vom SBV akkreditierte Ärzte zum Vertrag zu. Der SBV garantiert den Vertragsparteien MTK und FMH für die Qualität der zugelassenen Ärzte wie auch der von ihnen erbrachten Leistungen;

- liegt für die gemeinsame Kostensteuerung in der Verantwortung bei der FMH/SBV und MTK;

- $\quad$ wird bis spätestens 1. Januar 2006 eingeführt und löst auf dieses Datum die einschlägigen vertraglich vereinbarten Rahmenvertragsregelungen $a b$, denen bis dann auch die Belegärzte unterstehen.

Eine ähnliche Lösung wird auch hier mit den Krankenversicherern angestrebt, wobei die spezifischen Probleme des KVG-Bereichs berücksichtigt und das Verhältnis zu den Zusatzversicherungsverträgen geklärt werden muss. 